\title{
Aportes al conocimiento de la distribución y biología del estornino pinto (Sturnus vulgaris) en la provincia de La Pampa
}

\author{
Contributions to the Knowledge of European Starling (Sturnus \\ vulgaris) Distribution and Biology in La Pampa Province
}

María Soledad Liébana*1; Miguel Ángel Santillán²; Nicolás Peralta Seen²;

Miguel Fiorucci ${ }^{3}$; Jaime Bernardos'; Julieta Mallet ${ }^{1}$

1 Instituto de Ciencias de la Tierra y Ambientales de La Pampa (INCITAP)- CONICET/UNLPam. Mendoza 109, (6300) Santa Rosa, La Pampa, Argentina.

2 División Zoología, Museo de Historia Natural de La Pampa, Secretaría de Cultura, Gobierno de La Pampa, Pellegrini 180, (6300) Santa Rosa, La Pampa, Argentina.

3 Establecimiento "El Mirador", (6325) Naicó, La Pampa, Argentina

4 Estación Experimental INTA Anguil, Instituto Nacional de Tecnología Agropecuaria, Ruta 5 km 580, (6326) Anguil, La Pampa, Argentina.

* e-mail: soleliebana@hotmail.com

\section{RESUMEN}

El estornino pinto Sturnus vulgaris es una de las especies de aves más invasoras del mundo, causante de pérdidas económicas y de biodiversidad. En Argentina posee poblaciones establecidas y en expansión, y ya se han registrado interacciones negativas con especies nativas. Aquí se describe el estado actual de su distribución en la provincia de La Pampa, en la región central de Argentina, cuyo paisaje corresponde a dos provincias fitogeográficas que albergan una extensa comunidad de aves usuarias de huecos, susceptibles de verse perjudicadas por esta especie. Se presentan además el primer registro de cría en caja nido y la primera descripción de dieta de los pichones para el área. La información espacial se generó a partir de registros proveniente de bases de datos en línea y propios. En base a los resultados se presume que la expansión se estaría dando en dirección oeste desde las poblaciones iniciales en la provincia de Buenos Aires. La confirmación de cría exitosa, junto con la expansión en curso deben constituirse en una alerta ante el potencial riesgo para

Ref. bibliográfica: Liébana, M. S.; Santillán, M. A.; Peralta Seen, N.; Fiorucci, M.; Bernardos, J.; Mallet, J. 2020. "Aportes al conocimiento de la distribución y biología del estornino pinto (Sturnus vulgaris) en la provincia de La Pampa". Acta zoológica lilloana 64 (1): 43-57. Fundación Miguel Lillo, Tucumán, Argentina. D.O.I.: https://doi.org/10.30550/j.azl/2020.64.1/4

> Recibido: 4 de marzo de 2020 - Aceptado: 12 de mayo de 2020

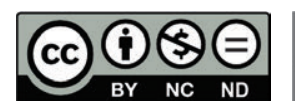


algunas especies usuarias de cavidades en caso de que las poblaciones prosperen. Resulta necesaria la implementación de monitoreos sistemáticos de la situación, para evaluar la eventual toma de medidas que mitiguen los potenciales efectos negativos sobre las aves nativas.

Palabras clave - Especie invasora, usuario de cavidades, dieta, reproducción, ciencia ciudadana.

\begin{abstract}
The European Starling Sturnus vulgaris is one of the most invasive bird species in the world, as it causes drastic economic and biodiversity losses. In Argentina, its populations are established and in expansion. Negative interactions of this species with native species have already been recorded. Here, we describe its current distribution in La Pampa province from central Argentina, whose landscape belongs to two phytogeographic provinces where an extensive community of cavity-nesting birds occur. Also, the first nest box breeding record and the first nestling's diet description is described for the region. Occurrence data were obtained from online databases of bird observations and from our own records. Based on these results, it is presumed that the expansion would be taking place in a western direction from the original populations in Buenos Aires province. Successful breeding confirmation, together with its ongoing expansion, should constitute an alert of the potential risk for some cavity user species. It is necessary to implement systematic surveys of the situation to assess the eventual execution of measures that mitigate the potential negative effects on native birds.
\end{abstract}

Keywords - Invasive species, cavity users, diet, reproduction, citizen science.

\title{
INTRODUCCIÓN
}

El estornino pinto Sturnus vulgaris es considerado una de las especies de aves más invasoras del mundo (Feare, 1984). Es nativa de Europa, oeste de Asia y norte de África (Feare, 1984) y actualmente, presenta la más amplia distribución a nivel global de todas las especies del género, con numerosas subespecies (Craig y Feare, 2009). Es una especie omnívora con un amplio espectro trófico, y que además varía su dieta durante las diferentes etapas de su ciclo de vida, consumiendo principalmente artrópodos durante la temporada reproductiva (Feare 1984). Entre los perjuicios que causa se encuentran las pérdidas asociadas a cultivos y a la cría de ganado (consumo de granos y pellets de alimento balanceado) (Hardy, 1990) y la pérdida de biodiversidad debido a la competencia con otras especies de aves, en particular con aquellas que nidifican en nidos cerrados o en cavidades, ya sean estas excavadas o naturales (Cabe, 1993; Koenig, 2003). El estornino pinto nidifica tanto en ambientes rurales como urbanos y puede utilizar cavidades artificiales, como huecos en estructuras 
de origen antrópico, e incluso cajas nido (Kessel, 1957; Moeed y Dawson, 1974; Lumsden, 1976; Feare 1984).

En Sudamérica, la especie se registra desde la década de 1980 y recientemente ha sido documentada en Uruguay y en Brasil (Peris, Soave, Camperi, Darrieu y Aramburu, 2005; Mazulla, 2013; Cavitione e Silva, da Motta Pinto, Mäder y de Souza, 2018). En Argentina no se ha estimado su tamaño poblacional (Ministerio de Ambiente y Desarrollo Sustentable y Aves Argentina, 2017) pero se encuentra en aumento al igual que su área de distribución (Peris et al., 2005; Zufiaurre, Abba, Bilenca y Codesido, 2016). Si bien sus poblaciones se consideran como bien establecidas en la región Pampeana (Zufiaurre et al., 2016) e incluso se han informado casos de uso de cavidades naturales y usurpación de nidos (Ibañez, Girini, Palacio, Fiorini, y Montalti, 2017), hasta el momento no se han corroborado efectos negativos significativos sobre las comunidades de aves nativas (Rebolo Ifrán y Fiorini, 2010; Palacio, Maragliano y Montalti, 2016). En cuanto a los potenciales perjuicios económicos, si bien se han registrado ataques a viñedos y frutales (Lucero, 2013; Zanotti, 2013; Ibañez, Andreucci y Montalti, 2016a) la magnitud de las pérdidas no ha sido evaluada. El perjuicio más notorio en Argentina es el provocado por las agregaciones en dormideros comunales de gran número de individuos en ciudades, las cuales generan malestar a los habitantes por los ruidos molestos y la acumulación de heces (Ibáñez, Zalba, Scorolli, Forcelli y Montalti, 2016b; Girini, Palacio, del Huerto y Kuzmanich, 2014).

Hasta el momento, la nidificación de esta especie ha sido extensamente documentada y estudiada en la provincia de Buenos Aires (Di Giacomo, Di Giacomo, y Barbaskas, 1993; Rivero, Lartigau, Caridad y Ramirez Llorens, 1996; Montalti y Kopij, 2001; Isacch e Isacch, 2004; Rebolo Ifrán y Fiorini, 2010; Rizzo, 2010; Di Sallo y Segura, 2014) y confirmada en las provincias de Córdoba (Klavins y Álvarez, 2012), Mendoza (Zanotti, 2013; Mendoza y Sorroche, 2020) y San Juan (Lucero, 2013). Mientras que la dieta ha sido solo estudiada por Ibañez (2015) a partir del análisis de contenidos estomacales de pichones en el noreste de la provincia de Buenos Aires. En cuanto a su demografía, se ha propuesto que la expansión poblacional sería en dirección este-oeste desde las poblaciones de la provincia de Buenos Aires, y que los centros urbanos actuarían como centros de dispersión (Zufiaurre et al. 2016).

La provincia de La Pampa, ubicada en la región central de Argentina y al oeste de la provincia de Buenos Aires, posee características paisajísticas que la hacen especialmente susceptible a la invasión por estornino pinto. Por un lado, el noreste de la provincia, perteneciente a la provincia fitogeográfica Pampeana (Pampa Interior Occidental) se caracteriza por una pronunciada actividad agrícola-ganadera (Oyarzabal et al., 2018), dónde en los últimos años se incrementó la cría de ganado bajo la modalidad de engorde en encierro (feed-lot). Por otro lado, se encuentra el Espinal, particularmente el distrito del Caldén, que limita con la provincia Pampeana. El paisaje en esta región se corresponde con un bosque semiárido compuesto principalmente por Caldén (Prosopis caldenia) (Cabrera, 1976). Este ambiente es hábitat de numerosas especies de aves, incluyendo 33 especies usuarias de cavidades distribuidas entre las diferentes categorías de uso; facultativo, parásito, obligada, casi obligado y excavador (López, 2014). La mayor parte de ellas podrían ser susceptibles de com- 
petencia con esta especie invasora, como por ejemplo los carpinteros (Colaptes campestris y Colaptes melanochloros; Ibañez et al., 2017, Jauregui 2020). Además, especies que construyen nidos cerrados también podrían ser afectadas por la usurpación por parte del estornino, como el Hornero (Furnarius rufus) (Aves Argentinas (AA), 1999; Narosky y Carman, 2009; Rizzo, 2010; Ibañez et al., 2017) o el Leñatero (Anumbius annumbi) (Di Sallo y Segura, 2014). Para la región central de Argentina y en particular para la provincia de La Pampa, no existen estudios publicados sobre la ecología trófica y reproductiva de la especie. En este trabajo se describen por primera vez el estado actual de su distribución en la provincia de La Pampa, basado en información proveniente de plataformas digitales y registros propios, el primer registro de cría en caja nido para la provincia y la dieta de los pichones.

\section{MATERIALES Y MÉTODOS}

El área de estudio corresponde a la provincia de La Pampa ubicada en la región central de Argentina, la cual comprende las provincias fitogeográficas del Espinal, del Monte y Pampeana (Oyarzabal et al., 2018). Los datos de distribución de la especie fueron obtenidos de las bases de datos en línea eBird Basic Dataset y EcoRegistros (eBird Basic Dataset, 2017; EcoRegistros, 2019). La búsqueda de datos se restringió al período comprendido entre los años 2013 (primer registro de la especie en la provincia) y 2019.

Además, se utilizaron registros propios obtenidos durante trabajos de campo en el área de estudio correspondientes a otros proyectos entre los años 2015 y 2019. La caja nido de la cual se obtuvo el registro de cría y los restos presa para determinar la dieta durante la cría de los pichones fue colocada a 5,5 m de altura, en un molino de viento de un establecimiento rural en cercanías de la localidad de Naicó dentro de la provincia fitogeográfica del Espinal (3650’30.43”S, 64²4'16.55”O) durante agosto del año 2017. El objetivo de esta era su ocupación por parte del Halconcito Colorado (Falco sparverius) y fue construida en madera siguiendo el diseño propuesto por Bortolotti (1994), con una altura frontal de $33 \mathrm{~cm}$, un ancho de $24 \mathrm{~cm}$ y una boca de entrada de $7,6 \mathrm{~cm}$ de diámetro ubicada a $26 \mathrm{~cm}$ del piso de la caja.

A partir de los restos presa encontrados en la caja nido cuando esta fue abandonada, se determinó la dieta de los pichones, la posible presencia de ectoparásitos y de fauna acompañante. El material fue analizado en laboratorio utilizando lupas binoculares, guías de referencia y material de referencia perteneciente a las colecciones del Museo de Historia Natural de La Pampa. Los restos presa fueron analizados siguiendo técnicas convencionales para la cuantificación de restos (Marti et al. 2007).

\section{RESULTADOS}

Recopilamos 48 registros de estornino pinto en la provincia de La Pampa (16 de origen propio, y 32 a las bases de datos eBird y EcoRegistros; Apéndice 1). Estos 
registros correspondieron a 18 localidades de la provincia de La Pampa (Apéndice 1). La mayor parte fue obtenida en localidades dentro de la provincia fitogeográfica Pampeana $(\mathrm{n}=41)$, principalmente en agroecosistemas del noreste de la provincia. Seis registros, incluyendo el registro de cría, corresponden a la provincia del Espinal, y solo uno a la del Monte (Figura 1). La mayor parte de los registros involucra uno o dos individuos; sin embargo, en dos ocasiones registramos bandadas, una de más de 50 individuos en un monte de Eucaliptus sp, y la otra de más de 200 individuos alimentándose en un lote de alfalfa (Medicago sativa). También encontramos un registro de una bandada con comportamiento de alimentación (EcoRegistros, 2018). Todos los casos de bandadas corresponden a la región agrícola-ganadera del noreste de la provincia.

En el año 2017 se constató la ocupación de la caja nido por parte de una pareja de la especie durante el mes de noviembre. El nido fue construido con restos vegetales y plumas de otras aves (Figura 2). El tamaño de puesta fue de seis huevos (25 de noviembre de 2017; Figura 2); el seis de diciembre de 2017 se hallaron cuatro pichones, mientras que a mediados de enero abandonaron el nido de manera exitosa dos volantones (Figura 3). No observamos adultos o volantones nuevamente en

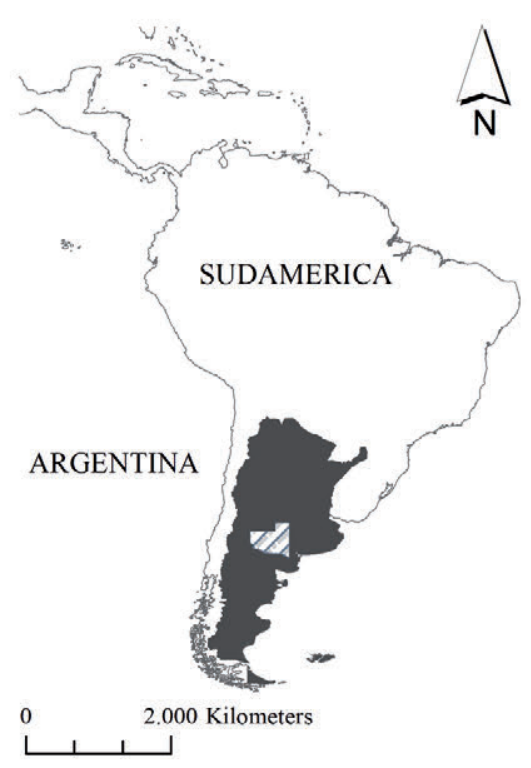

ECORREGION

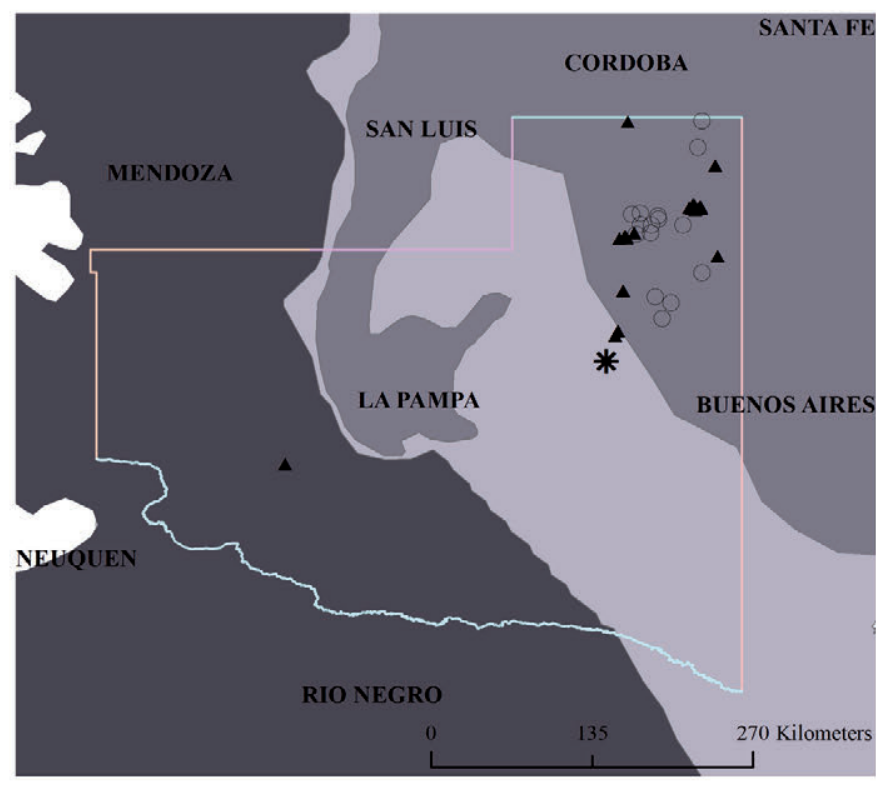

TIPOS DE REGISTROS

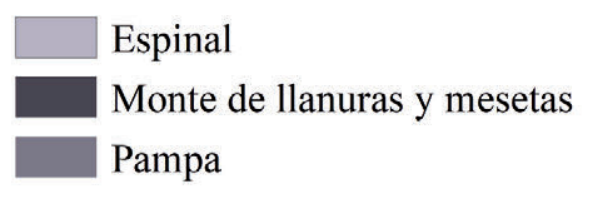

○ Registros propios

$\triangle$ Bases de datos

* Cría

Figura 1. Mapa de los registros de Estornino Pinto en la provincia de La Pampa, Argentina (años 2013 a 2018).

Figure 1. Map of the records of European Starling in the province of La Pampa, Argentina (years 2013 to 2018). 
las inmediaciones del nido. Al año siguiente, la caja fue ocupada durante el mes de octubre por Halconcito Colorado y criaron exitosamente.

En cuanto a la dieta, se analizaron en total 838 restos presa, los cuales presumiblemente corresponden a las presas consumidas por los pichones durante el período de cría. Se hallaron representados en la dieta dos clases y siete órdenes de artrópodos (Tabla 1). Los insectos fueron identificados y cuantificados a partir de cabezas o pares de mandíbulas y élitros, mientras que los arácnidos lo fueron a partir de pares quelíceros. La mayor parte de los ítems estuvo representada por ortópteros, en un valor mayor al 50\%, seguidos por coleópteros en cerca de un $40 \%$ (adultos y larvas). Finalmente, en mucha menor medida también consumieron otros órdenes de las clases Insecta y Arachnida (Tabla 1). También se encontraron entre los restos presa pequeños fragmentos de fósiles $(\mathrm{n}=10)$ y piedras $(\mathrm{n}=44)$.

Dentro del nido encontramos los ectoparásitos Ornithonyssus bursa (Acari: Mesostigmata; $\mathrm{n}=3$ ) y Taphropiestes plaumanni (Coleoptera: Cavognathidae; $\mathrm{n}=30$ ) que habrían estado parasitando a los individuos, e individuos del orden de los pseudoescorpiones (Arachnida: Pseudoscorpiones; $\mathrm{n}=4$ ).

\section{DISCUSIÓN}

El primer registro de estornino pinto en Argentina es de 1987 (Pérez, 1988) en la provincia de Buenos Aires. Sin embargo, ya en 1983 se habrían liberado individuos en la zona ribereña de Punta Lara, provincia de Buenos Aires (Navas, 2002). Para la provincia de La Pampa el primer registro documentado en una base de datos ocurrió en el año 2013 en Santa Rosa (eBird, 2017), ciudad que se encuentra en el ecotono entre las provincias fitogeográficas Pampeana y del Espinal. El origen de los individuos observados en el área de estudio es incierto. Si bien podría tratarse de la expansión natural de la especie desde los sitios ya colonizados, también existe

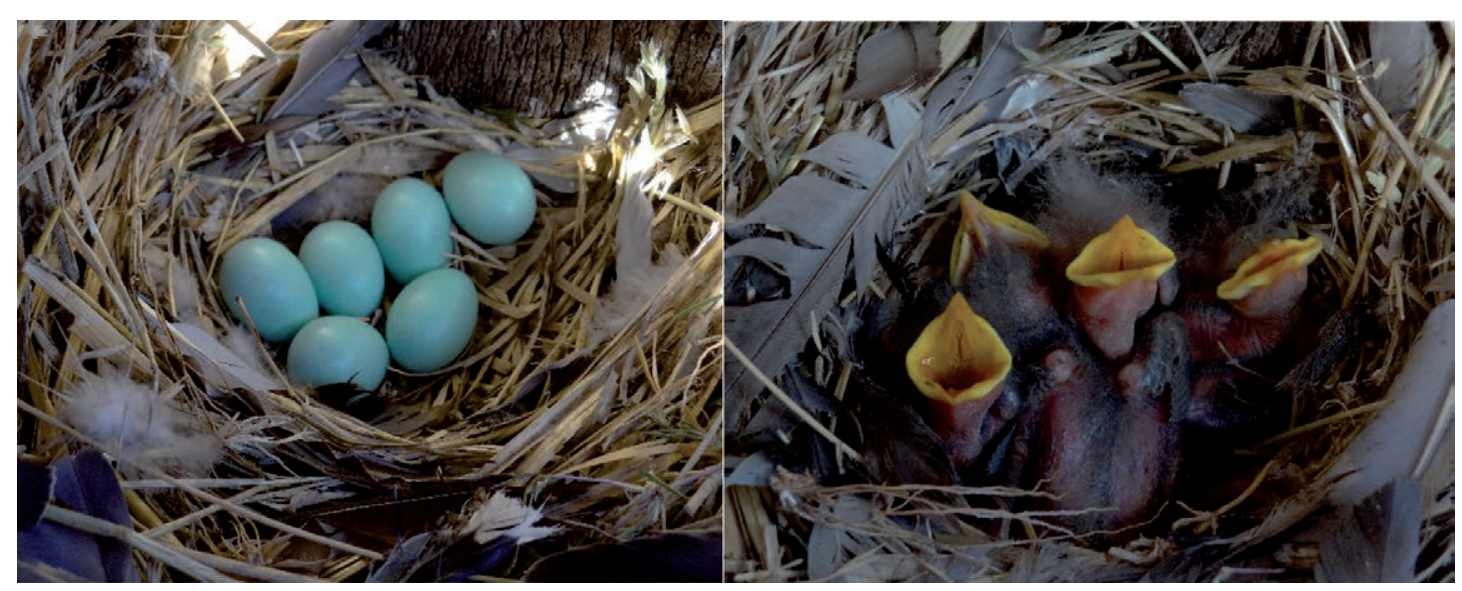

Figura 2. Huevos y pichones de Estornino Pinto criando en caja nido en la provincia fitogeográfica del Espinal, provincia de La Pampa, Argentina.

Figure 2. Eggs and chicks of European Starling breeding in nest box in the phytogeographic province of Espinal, La Pampa province, Argentina. 
Tabla 1. Presas consumidas por pichones del Estornino Pinto en la provincia fitogeográfica del Espinal, La Pampa, Argentina. $\mathrm{N}=$ número de restos presa; $\mathrm{P}=$ porcentaje de ocurrencia; $\mathrm{NI}=$ familia no identificada.

Table 1. Prey consumed by European Starling chicks in the phytogeographic province of Espinal, La Pampa province, Argentina. $\mathrm{N}=$ number of preys remains; $\mathrm{P}=$ occurrence percentage; $\mathrm{NI}=$ unidentified family.

\begin{tabular}{|c|c|c|c|}
\hline Orden & Familia & $N$ & $P(\%)$ \\
\hline \multicolumn{4}{|l|}{ Orthoptera } \\
\hline & Acrididae & 390 & 46,5 \\
\hline & Tettigonidae & 22 & 2,6 \\
\hline & Grillydae & 12 & 1,4 \\
\hline Mantodea & & 6 & 0,7 \\
\hline \multirow[t]{3}{*}{ Coleoptera } & Larvas NI & 226 & 27,0 \\
\hline & Adultos NI & 82 & 9,8 \\
\hline & Curculionidae & 18 & 2,1 \\
\hline \multirow[t]{3}{*}{ Hemiptera } & Hemiptera NI & 1 & 0,1 \\
\hline & Rhopalidae & 2 & 0,2 \\
\hline & Pentatomidae & 1 & 0,1 \\
\hline \multirow[t]{3}{*}{ Hymenoptera } & Hymenoptera NI & 7 & 0,8 \\
\hline & Formicidae & 13 & 1,6 \\
\hline & & & 0 \\
\hline Scorpiones & Bothriuridae & 24 & 2,9 \\
\hline \multicolumn{4}{|l|}{ Araneae } \\
\hline & Lycosidae & 34 & 4,1 \\
\hline Total & & 838 & \\
\hline
\end{tabular}

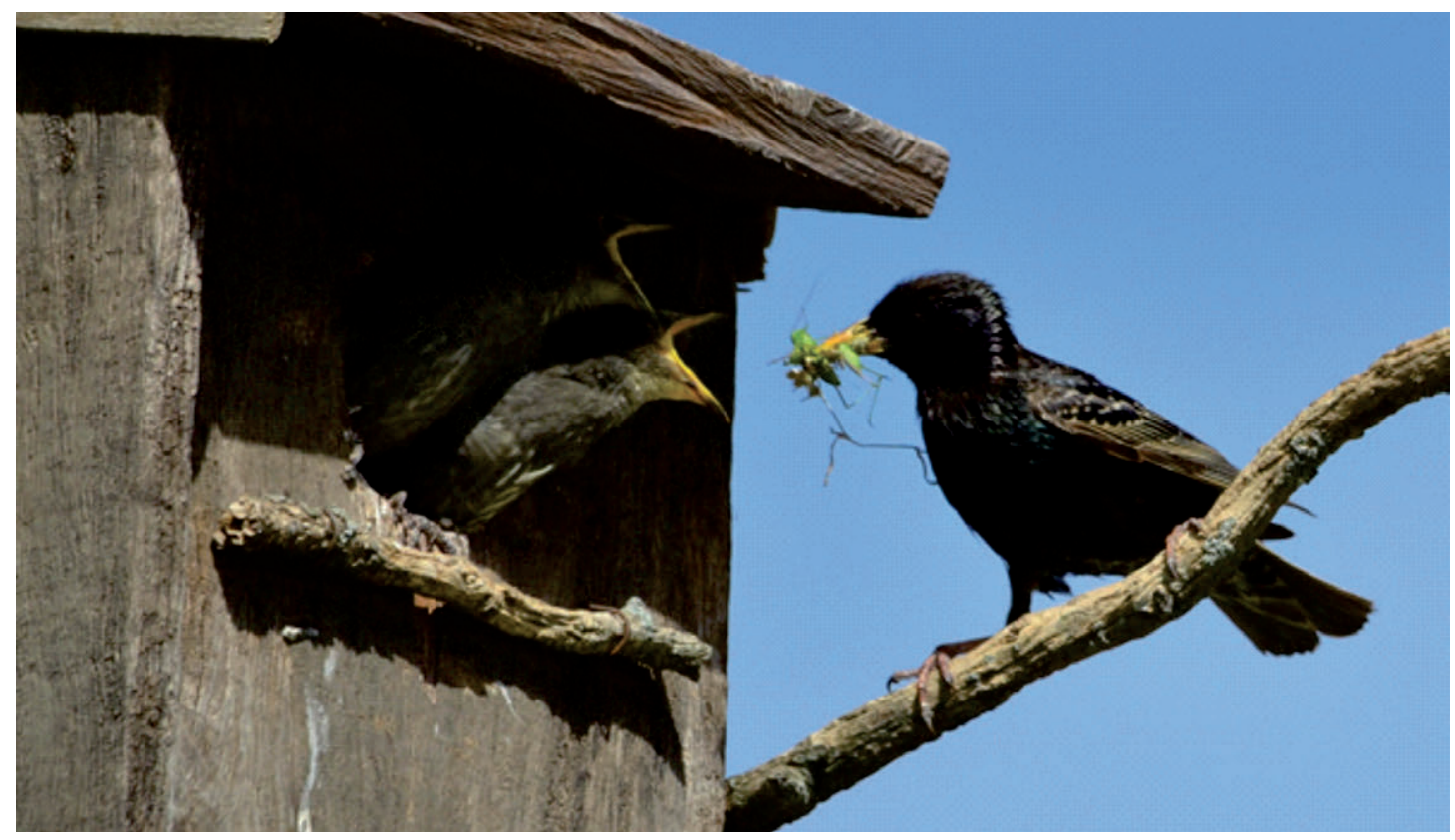

Figura 3. Adulto de Estornino Pinto alimentando dos pichones en caja nido, en la provincia fitogeográfica del Espinal, provincia de La Pampa, Argentina.

Figure 3. Adult of European Starling feeding two nestlings in a nest box, in the phytogeographic province of Espinal, La Pampa province, Argentina. 
el tráfico ilegal de individuos de la especie en la provincia (datos no publicados de la Dirección de Recursos Naturales de La Pampa), cuyos posibles escapes podrían actuar como potenciador de la expansión natural. Se ha propuesto que las poblaciones de las provincias de Santa Fe y Entre Ríos, así como aquellas del SE de la provincia de Buenos Aires, tuvieron un origen diferente a las del NE bonaerense (Jensen, 2008) y que las mismas podrían provenir de otras liberaciones (Navas, 2002). Teniendo en cuenta los registros observados en las plataformas digitales más los datos publicados, cabría esperar que el ingreso a la provincia de La Pampa de la especie haya ocurrido desde los límites este con la provincia de Buenos Aires y norte con la provincia de Córdoba, en concordancia con el avance en dirección oeste desde la provincia de Buenos Aires, siguiendo la expansión observada por Zufiaurre et al. (2016).

La presencia de Estornino en áreas rurales también se relaciona de manera positiva con la cercanía de pequeños centros urbanos como estaciones de trenes o casas rurales, que actuarían como centros de dispersión hacia localidades adyacentes (Zufiaurre et al., 2016). En concordancia con esto, el sitio de nidificación documentado en este trabajo se halla en el casco de un establecimiento rural, aunque de un paisaje de bosque de Caldén, notoriamente diferente de la región pampeana. Por otro lado, solo se trata de una pareja y no de una población establecida, lo cual no permite realizar mayores conjeturas. Como se comentó anteriormente, uno de los registros corresponde a una bandada que utilizaba una arboleda de especies exóticas (Eucaliptus sp.) como dormidero. El uso de árboles exóticos por el estornino ya ha sido documentado (Carro, Lacoretz y Acosta, 2012; Girini et al. 2014) y resulta de especial relevancia en zonas agrícolas, donde este elemento fisonómico se introdujo junto con el resto de las trasformaciones agrícolas durante el siglo pasado. El eucaliptus en particular actuó históricamente como facilitador para la expansión de aves nativas como la cotorra (Myiopsitta monachus) en la provincia fitogeográfica pampeana (Bucher y Aramburu, 2014), por lo cual podría actuar como otro elemento facilitador para la expansión del estornino. A su vez, Zufiaurre et al. (2016) determinaron que los asentamientos humanos pequeños, los cuales la mayoría se hayan rodeados de arboledas, actúan como proveedores de recursos para nidificar y alimentarse.

El uso de cajas nido destinadas a la especie Halconcito Colorado ya ha sido descripta y estudiada en Norteamérica (Valdez, Robertson, Robertson y Bildstein, 2000) y se ha propuesto que los halconcitos son capaces de competir exitosamente con los estorninos por el uso de cavidades y cajas nido (Bechard y Bechard, 1996). Si bien al año siguiente de la ocupación de la caja por estorninos (año 2018), una pareja de Halconcito Colorado crio de manera exitosa, no pudimos determinar si existió competencia. Ibañez (2015) estudió distintos aspectos de la historia natural (cría, dieta y parásitos) de la especie a partir del uso de cajas nido en la costa del Río de la Plata, siendo consideradas estas como una potencial herramienta para el control de las poblaciones silvestres (Campbell et al., 2016). Los resultados de puesta y de éxito reproductivo coinciden con lo reportado por Ibáñez (2015). El escenario podría ser diferente cuando se trata de competencia por el uso de cavidades naturales con otras especies. Por ejemplo, es conocido el desplazamiento de parejas reproductoras de carpinteros (Colpates spp.) (Rebolo Ifrán y Fiorini, 2010; Ibañez et al. 2017; Jauregui 2020).

La dieta de los pichones para el área de estudio no difiere de la ya determinada 
para la especie, siendo principalmente insectívora durante la época de cría (Feare, 1984; Cabe, 1993) y es similar a la determinada por Ibañez (2015) en cuanto al elevado consumo de coleópteros (tanto larvas como adultos). Sin embargo, en este estudio las presas principales fueron los ortópteros, y en particular los acrídidos, lo cual podría estar explicado por el carácter oportunista de la especie, al consumir un tipo de presa altamente disponible en el ambiente (Bardi, 2013). En este estudio se analizaron los restos presa presentes en la caja nido, con lo cual no fue posible determinar el grado de consumo de material vegetal, el cual no fue hallado entre los restos. Los pequeños fósiles hallados entre los restos presa podrían haber sido ingeridos de manera similar a las piedras encontradas por Ibañez (2015) en estómagos de pichones de estornino pinto. Este comportamiento se halla muy generalizado entre las aves y se han propuesto diversas hipótesis explicativas, como por ejemplo las que lo relacionan con una facilitación de la digestión de material vegetal o a un aporte de minerales esenciales entre otras (Downs, Bredin y Wragg, 2019)

Los artrópodos encontrados en el nido son de particular relevancia. Por un lado, Taphropiestes plaumanni es un coleóptero registrado en diferentes nidos de aves (Rebollo, 2015; Di Iorio y Turienzo, 2016), pero es la primera vez registrado en un nido de estornino pinto. Por otro lado, el ácaro Ornithonyssus bursa es una especie hematófaga que ha ya sido documentada en cajas nido de estornino pinto, así como también parasitando aves de la región central de Argentina (Arrabal, Manzoli, Antoniazzi, Lareschi y Beldomenico, 2012; Ibañez, 2015; Santillán et al. 2015; Lareschi, Cicuttin, Salvo, Ibañez y Montalti, 2017). Por ejemplo, Ibañez (2015) halló que el $97,5 \%$ de los pichones se hallaban parasitados por la especie $(n=79)$. Los pseudoescorpiones también han sido registrados en nidos de Estornino en Nueva Zelanda y en países de Europa (Turienzo, Di Iorio y Mahnert, 2010). Sin embargo, en Argentina sólo ha sido hallado en nidos de Cotorra, Leñatero (Anummbi anummbi) y Espinero (Phacellodomus ruber) (Mahnert, Turienzo y Di Iorio, 2011).

La confirmación de la cría exitosa por parte del Estornino pinto en el Espinal, junto con la expansión en curso en la región deben constituirse en una alerta ante el potencial riesgo al que se podrían enfrentar al menos algunas especies usuarias de cavidades en caso de que las poblaciones prosperen. Resulta necesario la implementación de monitoreos sistemáticos de la situación en los diversos ambientes de la provincia, para determinar consecuentemente la necesidad de puesta en marcha de medidas que tiendan a mitigar los potenciales efectos negativos sobre las poblaciones de aves nativas.

\section{AGRADECIMIENTOS}

A todas las personas que a través del uso de bases de datos en línea (eBird, EcoRegistros) contribuyen al desarrollo de la Ciencia Ciudadana. Agradecemos a los dos revisores anónimos y editores que con sus correcciones y sugerencias mejoraron notablemente el manuscrito. 


\section{FINANCIAMIENTO}

Las muestras colectadas fueron depositadas en el Museo de Historia Natural de La Pampa. No se contó con financiamiento específico para este trabajo.

\section{PARTICIPACIÓN}

MSL Y MAS participaron en los muestreos de campo, análisis de datos y redacción del manuscrito. NPS y MAS realizaron el análisis de la dieta, MF realizó el monitoreo de la actividad en la caja nido, y JB y JM participaron en la toma de datos y redacción del manuscrito.

\section{CONFLICTOS DE INTERÉS}

No existen conflictos de interés entre autores o con terceros.

\section{LITERATURA CITADA}

Arrabal, J.P., Manzoli, D.E., Antoniazzi, L.R., Lareschi, M., Beldomenico, P.M. (2012). Prevalencia del ácaro Ornithonyssus bursa Berlese, 1888 (Mesostigmata: Macronyssidae) en un ensamble de aves (Passeriformes) de bosques del centro de la provincia de Santa Fé, Argentina. Revista Ibero-Latinoamericana de Parasitología, 71, 172-178.

Aves Argentinas. (1999). Campaña de estudio de los estorninos introducidos en la República Argentina. Aves Argentinas/Asociación Ornitológica del Plata, Buenos Aires, Argentina.

Bardi, C.J. (2013). Biología y biocontrol de Dichroplus elongatus Giglio-Tos (Orthoptera: Acrididae: Melanoplinae), acridio plaga del agro en Argentina. Tesis doctoral. Facultad de Ciencias Naturales y Museo, Universidad Nacional de La Plata. http://naturalis.fcnym.unlp.edu.ar/id/20130909001297

Bechard, M.J., Bechard J.M. (1996). Competition for nest boxes between American Kestrels and European Starlings in an agricultural area of southern Idaho. En Raptors in human landscapes: adaptation to built and cultivated environments (155 - 162). Academic Press, London.

Bucher, E.H., Aramburu, R.M. (2014). Land-use changes and monk parakeet expansion in the Pampas grasslands of Argentina. Journal of Biogeography, 41, 1160-1170. https://doi.org/10.1111/jbi.12282

Bortolotti, G.R. (1994). Effect of nest-box size on Nest-Site preference and reproduction in American Kestrels. Journal of Raptor Research, 28(3), 127-133.

Cabe, P.R. (1993). European Starling (Sturnus vulgaris), version 2.0. En The Birds of North America. Cornell Lab of Ornithology, Ithaca, NY, USA. 
Cabrera, A.L. 1976. Regiones fitogeográficas argentinas. Enciclopedia Argentina de Agricultura y Jardinería. Editorial ACME.

Carro, M.E., Lacoretz, M.V., Acosta, T.H. (2014). El estornino pinto (Sturnus vulgaris) en General Lavalle, Buenos Aires, Argentina. Nuestras Aves, 59, 29-30.

Campbell, S., Roberts, E.J., Craemer, R., Pacioni, C., Rollins, L., Woolnough, A.P. (2016). Assessing the economic benefits of starling detection and control to Western Australia. Australasian Journal of Environmental Management, 23, 81-99. https://doi.org/10.1080/14486563.2015.1028486

Cavitione e Silva, F., da Motta Pinto, J., Mäder, A., de Souza, V.A.T. (2018). First records of European Starling Sturnus vulgaris in Brazil. Revista Brasileira de Ornitologia-Brazilian Journal of Ornithology, 25, 297-298. https://doi.org/10.1007/ BF03544409

Craig, A.J., Feare, C.J. (2009). Family Sturnidae (starlings). En Handbook of the birds of the world, Vol.14. Bush-shrikes to Old World sparrows (654-758). Lynx Editions, Barcelona.

Downs, C.T., Bredin, I.P., Wragg, P.D. (2019). More than eating dirt: a review of avian geophagy. African Zoology, 54(1), 1-19. 10.1080/15627020.2019.1570335

Di Iorio, O.D., Turienzo P. (2016). The family Cavognathidae (Coleoptera: Cucujoidea) in Argentina and adjacent countries. Zootaxa, 4092, 90-106. 10.11646/zootaxa.4092.1.5

Di Giacomo, A.G., Di Giacomo, A.S., Barbaskas, M. (1993). Nuevos registros de Sturnus vulgaris y Acridotheres cristatellus en Buenos Aires. Nuestras Aves, 29, 2-3.

Di Sallo, F.G, Segura, L.N. (2014). Nidificación del estornino pinto (Sturnus vulgaris) en un nido de leñatero (Anumbius annumbi) en el noreste de la provincia de Buenos Aires, Argentina. Nuestras Aves, 59, 13-15.

eBird Basic Dataset. (2017). Versión: EBD_relNov-2017. Cornell Lab of Ornithology, Ithaca, New York.

EcoRegistros (2019). estornino pinto en la provincia de La Pampa, Argentina. Accedido de http://www.ecoregistros.org.

Feare C. J. (1984). The starling. Oxford University Press, Oxford.

Girini, J.M., Palacio, F.X., del Huerto, M.D.C., Kuzmanich, N. (2014). Selección de dormideros por el estornino pinto (Sturnus vulgaris) en La Plata, Buenos Aires, Argentina. Hornero, 29, 23-28.

Hardy, A.R. (1990). Vertebrate pests of UK agriculture: present problems and future solutions. En Proceedings of the Vertebrate Pest Conference, 14, 14.

Ibañez, L.M. (2015). Invasión del estornino pinto Sturnus vulgaris en el Noreste de la provincia de Buenos Aires: análisis de la competencia con aves nativas y potencialidad como transmisor de parásitos. Tesis Doctoral. Facultad de Ciencias Naturales y Museo, Universidad Nacional de La Plata, Buenos Aires, Argentina.

Ibañez, L.M., Girini, J.M., Palacio, F.X., Fiorini, V.D., Montalti, D. (2017). Interacciones entre el estornino pinto (Sturnus vulgaris) y aves nativas de Argentina por el uso de cavidades. Revista mexicana de biodiversidad, 88: 477-479. 10.1016/ j.rmb.2017.03.009

Ibañez, L.M., Andreucci, F., Montalti, D. (2016a). Primer registro de daño a cultivo de frutales por el estornino pinto (Sturnus vulgaris) (Passeriformes: Sturnidae) en Argentina. Acta zoológica lilloana, 60, 177-180. 
Ibañez, L.M., Zalba, S.M., Scorolli, A., Forcelli, D., Montalti, D. (2016b). El estornino pinto en Argentina: desafíos y prioridades. Revista del Museo de La Plata, 1.

Isacch, J.P., Isacch, J. (2004). Estornino pinto (Sturnus vulgaris) en la ciudad de Mar del Plata (Buenos Aires, Argentina). Nuestras Aves, 47, 33.

Jauregui, A. (2020) Selección de sitios de nidificación y efecto del hábitat en el éxito reproductivo de Colaptes campestris (Carpintero Campestre) y Colaptes melanochloros (Carpintero Real) (Aves: Picidae) en talares bonaerenses. Tesis Doctoral. Universidad Nacional de La Plata.

Jensen, F.R. (2008). Nuevos registros de estornino pinto (Sturnus vulgaris) para el sureste de la provincia de Entre Ríos, Argentina. Nuestras Aves, 53, 22.

Kessel, B. (1957). A study of the breeding biology of the European Starling (Sturnus vulgaris L.) in North America. The American Midland Naturalist, 58, 257-331.

Klavins, J. y Álvarez, D. (2012). El estornino pinto (Sturnus vulgaris) en la provincia de Córdoba, Argentina. Nuestras Aves, 57, 27-29.

Koenig, W.D. (2003). European starlings and their effect on native cavity nesting birds. Conservation Biology, 17, 1134-1140. 10.1046/j.1523-1739.2003.02262.x

Lareschi, M., Cicuttin, G.L., Salvo, M.N.D., Ibañez, L., Montalti, D. (2017). El ácaro de aves tropicales Ornithonyssus bursa (Acari: Mesostigmata: Macronyssidae) parasitando al estornino pinto Sturnus vulgaris (Aves: Passeriformes: Sturnidae), un ave invasiva en el centro de Argentina. Una aproximación a la fauna bacteriana de este ácaro. Revista mexicana de biodiversidad, 88, 454-458. http://dx.doi. org/10.1016/j.rmb.2017.03.022

López, F.G. (2014). Oferta de cavidades para vertebrados en relación a parámetros de sustrato de bosques en distinto grado de estado sucesional en el caldenal pampeano. Tesis de grado. Universidad Nacional de La Pampa, La Pampa, Argentina.

Lucero, F. (2013). Nuevas aves, primeras evidencias y localidades para las provincias de San Juan y Catamarca, Argentina. Ecoregistros, 3, 14.

Lumsden, H.G. (1976). Choice of nest boxes by Starlings. The Wilson Bulletin, 88, 665-666.

Mazulla, J. (2013). Primeros registros de estornino pinto Sturnus vulgaris (Linnaeus, 1758) (Aves, Passeriformes, Sturnidae) en Uruguay. Revista Achará, 3, 13-17.

MAyDS y AA (Ministerio de Ambiente y Desarrollo Sustentable y Aves Argentina) (2017). Categorización de las Aves de la Argentina (2015). Ministerio de Ambiente y Desarrollo Sustentable de la Nación y de Aves Argentinas, edición electrónica. C. A. Buenos Aires, Argentina.

Mahnert, V., Turienzo, P., Di Iorio, O. (2011). Pseudoscorpions (Arachnida) from Argentina. New records of distributions and habitats, corrections and an identification key. Zootaxa, 2881, 1-30. 10.11646/zootaxa.2881.1.1

Mendoza, F., Sorroche, S. (2020). Evidencias de nidificación de estornino pinto (Sturnus vulgaris) en la provincia de Mendoza, Argentina. Nótulas Faunísticas - Segunda Serie, 289, 1-5. https://www.fundacionazara.org.ar/img/notulas-faunisticas/articulos/notula-289.pdf 
Moeed, A., Dawson, D.G. (1979). Breeding of Starlings (Sturnus vulgaris) in nest boxes of various types. New Zealand Journal of Zoology, 6, 613-618. 10.1080/0 3014223.1979.10428404

Montalti, D., Kopij, G. (2001). Bird community of inner La Plata city, Argentina. Acta Ornithologica, 36, 161-164. https://doi.org/10.3161/068.036.0209

Narosky T., Carman, R.L. (2009). El hornero ave nacional. Editorial Albatros: Buenos Aires, Argentina.

Navas, J.R. (2002). Las aves exóticas introducidas y naturalizadas en la Argentina. Revista del Museo Argentino de Ciencias Naturales, 4, 191-202.

Oyarzabal, M., Clavijo, J. R., Oakley, L. J., Biganzoli, F., Tognetti, P.M., Barberis, I.M., Maturo, H.M., Aragón, R., Campanello, P.I., Prado, D., Oesterheld, M. (2018). Unidades de vegetación de la Argentina. Ecología Austral, 28, 040-063. http://dx.doi.org/10.25260/EA.18.28.1.0.399

Palacio, F.X., Maragliano, R.E., Montalti, D. (2016). Functional role of the invasive European Starling, Sturnus vulgaris, in Argentina. Emu, 116, 387-393. https:// dx.doi.org/10.1071/MU16021

Pérez, J. (1988). Estornino pinto en la Capital Federal. Nuestras Aves. 17, 14.

Peris, S., Soave, G., Camperi, A., Darrieu, C., Aramburu, R. (2005). Range expansion of the European Starling Sturnus vulgaris in Argentina. Ardeola, 52, 359-364.

Rebolo Ifrán, N., Fiorini, D.V. (2010). European starling (Sturnus vulgaris): population density and interactions with native species in Buenos Aires urban parks. Ornitología Neotropical, 21, 507-518.

Rebollo, M.E. (2015). Biología reproductiva del Coludito copetón (Leptasthenura platensis) en un fragmento de bosque de caldén, La Pampa. Tesina de Licenciatura. Universidad Nacional de La Pampa, La Pampa, Argentina.

Rivero, E.M., Lartigau, B., Caridad, P.H., Ramirez Llorens, P. (1996) Registro concreto de nidificación del estornino pinto (Sturnus vulgaris) en la Argentina. Nuestras Aves, 34, 46.

Rizzo, F. (2010). Utilización de nidos de Hornero (Furnarius rufus) por el estornino pinto (Sturnus vulgaris). Nuestras Aves, 55, 33-35.

Santillán, M. A., Grande, J.M., Liébana, M.S., Martínez, P., Díaz, L.A., Bragagnolo, L.A., Solaro, C., Galmes, M.A., Sarasola, J.H. (2015). New hosts for the mite Ornithonyssus bursa in Argentina. Medical and Veterinary Entomology, 29, 439443. $10.1111 / \mathrm{mve} .12129$

Turienzo, P., Di Iorio, O., Mahnert, V. (2010). Global checklist of pseudoscorpions (Arachnida) found in birds' nests. Revue suisse de Zoologie, 117, 557-598.

Valdez, U., Robertson, S., Robertson, B., Bildstein, K.L. (2000). Nestbox use by American kestrels (Falco sparverius) and European starlings (Sturnus vulgaris) in eastern Pennsylvania. Pennsylvania Birds, 14, 150-153.

Zanotti, M. (2013). Presencia del estornino pinto (Sturnus vulgaris) en la provincia de Mendoza, Argentina. Nuestras Aves, 58, 5-7.

Zufiaurre, E., Abba, A., Bilenca, D., Codesido, M. (2016). Role of landscape elements on recent distributional expansion of European Starlings (Sturnus vulgaris) in agroecosystems of the Pampas, Argentina. The Wilson Journal of Ornithology, 128, 306-313. https://doi.org/10.1676/wils-128-02-306-313.1 
Apéndice 1. Registros de Estornino Pinto en la provincia de La Pampa, Argentina (años 2013 a 2019; $\mathrm{n}=$ número de individuos observados).

Appendix 1. Records of European Starling in the province of La Pampa, Argentina (years 2013 to 2019; $n=$ number of individuals observed).

\begin{tabular}{|c|c|c|c|c|c|c|}
\hline ID & $\begin{array}{l}\text { Fecha } \\
\text { registro }\end{array}$ & $\mathbf{n}$ & $\begin{array}{l}\text { Coordenadas } \\
\text { geográficas }\end{array}$ & $\begin{array}{l}\text { Localidad } \\
\text { más cercana }\end{array}$ & $\begin{array}{l}\text { Eco- } \\
\text { región }\end{array}$ & Fuente \\
\hline \multirow[t]{2}{*}{ SV1 } & $13 / 10 / 2018$ & 2 & $35^{\circ} 44^{\prime} 2.29^{\prime \prime} \mathrm{S}$ & Trenel & Pampa & Propio \\
\hline & & & $64^{\circ} 12^{\prime} 58.18^{\prime \prime O}$ & & & \\
\hline \multirow[t]{2}{*}{ SV2 } & $13 / 10 / 2018$ & 4 & $35^{\circ} 44^{\prime} 48.77^{\prime \prime} \mathrm{S}$ & Trenel & Pampa & Propio \\
\hline & & & $64^{\circ} 0^{\prime} 55.55^{\prime \prime O}$ & & & \\
\hline \multirow[t]{2}{*}{ SV3 } & $13 / 10 / 2018$ & 2 & $35^{\circ} 48^{\prime} 48.17^{\prime \prime S}$ & Trenel & Pampa & Propio \\
\hline & & & $64^{\circ} 3^{\prime} 54.00^{\prime \prime} \mathrm{O}$ & & & \\
\hline \multirow[t]{2}{*}{ SV4 } & $10 / 10 / 2015$ & 3 & $35^{\circ} 1^{\prime} 41.29^{\prime \prime} \mathrm{S}$ & Sarah & Pampa & Propio \\
\hline & & & $63^{\circ} 41^{\prime} 9.56^{\prime \prime} \mathrm{O}$ & & & \\
\hline \multirow[t]{2}{*}{ SV5 } & $16 / 11 / 2016$ & 2 & $35^{\circ} 53^{\prime} 3.39^{\prime \prime} \mathrm{S}$ & Monte Nievas & Pampa & Propio \\
\hline & & & $64^{\circ} 10^{\prime} 52.92^{\prime \prime O}$ & & & \\
\hline \multirow[t]{2}{*}{ SV6 } & $16 / 11 / 2016$ & $\geq 50$ & $35^{\circ} 52^{\prime} 29.29^{\prime \prime} \mathrm{S}$ & Monte Nievas & Pampa & Propio \\
\hline & & & $64^{\circ} 4^{\prime} 24.52^{\prime \prime} \mathrm{O}$ & & & \\
\hline \multirow[t]{2}{*}{ SV7 } & $25 / 11 / 2017$ & 2 & $36^{\circ} 50^{\prime} 30.43^{\prime \prime S}$ & Naicó & Espinal & Propio \\
\hline & & & $64^{\circ} 24^{\prime} 16.55^{\prime \prime O}$ & & & \\
\hline \multirow[t]{2}{*}{ SV8 } & $19 / 07 / 2017$ & 1 & $35^{\circ} 21^{\prime} 48.89^{\prime \prime} \mathrm{S}$ & Intendente Alvear & Pampa & e-Bird \\
\hline & & & $63^{\circ} 34^{\prime} 58.97^{\prime \prime} \mathrm{O}$ & & & \\
\hline \multirow[t]{2}{*}{ SV8bis } & $16 / 08 / 2018$ & 3 & $35^{\circ} 21^{\prime} 48.89^{\prime \prime} \mathrm{S}$ & Intendente Alvear & Pampa & e-Bird \\
\hline & & & $63^{\circ} 34^{\prime} 58.97^{\prime \prime O}$ & & & \\
\hline \multirow[t]{2}{*}{ SV9 } & $31 / 07 / 2018$ & 2 & $35^{\circ} 41^{\prime} 23.99^{\prime \prime} \mathrm{S}$ & General Pico & Pampa & e-Bird \\
\hline & & & $63^{\circ} 41^{\prime} 31.17^{\prime \prime} \mathrm{O}$ & & & \\
\hline \multirow[t]{2}{*}{ SV10 } & $27 / 05 / 2015$ & 58 & $35^{\circ} 41^{\prime} 56.35^{\prime \prime} \mathrm{S}$ & General Pico & Pampa & e-Bird \\
\hline & & & $63^{\circ} 44^{\prime} 7.26^{\prime \prime} \mathrm{O}$ & & & \\
\hline \multirow[t]{2}{*}{ SV11 } & $04 / 11 / 2017$ & 3 & $35^{\circ} 41^{\prime} 17.25^{\prime \prime S}$ & General Pico & Pampa & e-Bird \\
\hline & & & $63^{\circ} 45^{\prime} 27.30^{\prime \prime} \mathrm{O}$ & & & \\
\hline \multirow[t]{2}{*}{ SV12 } & $28 / 05 / 2015$ & 1 & $35^{\circ} 41^{\prime} 25.22^{\prime \prime S}$ & General Pico & Pampa & e-Bird \\
\hline & & & $63^{\circ} 45^{\prime} 32.54^{\prime \prime O}$ & & & \\
\hline \multirow[t]{2}{*}{ SV13 } & 29/08/2018 & 1 & $35^{\circ} 40^{\prime} 44.40^{\prime \prime} \mathrm{S}$ & General Pico & Pampa & e-Bird \\
\hline & & & $63^{\circ} 42^{\prime} 10.80^{\prime \prime O}$ & & & \\
\hline \multirow[t]{2}{*}{ SV14 } & $06 / 10 / 2018$ & 2 & $35^{\circ} 40^{\prime} 44.40^{\prime \prime} \mathrm{S}$ & General Pico & Pampa & e-Bird \\
\hline & & & $63^{\circ} 41^{\prime} 38.40^{\prime \prime} \mathrm{O}$ & & & \\
\hline \multirow[t]{2}{*}{ SV15 } & $19 / 09 / 2013$ & 1 & $36^{\circ} 37^{\prime} 7.80^{\prime \prime} \mathrm{S}$ & Santa Rosa & Espinal & e-Bird \\
\hline & & & $64^{\circ} 19^{\prime} 15.61^{\prime \prime O}$ & & & \\
\hline \multirow[t]{2}{*}{ SV16 } & $31 / 10 / 2018$ & 1 & $35^{\circ} 40^{\prime} 38.87^{\prime \prime S}$ & General Pico & Pampa & Eco-Registros \\
\hline & & & $63^{\circ} 44^{\prime} 21.04^{\prime \prime O}$ & & & \\
\hline \multirow[t]{2}{*}{ SV17 } & $16 / 09 / 2018$ & 1 & $35^{\circ} 39^{\prime} 50.37^{\prime \prime S}$ & General Pico & Pampa & Eco-Registros \\
\hline & & & $63^{\circ} 44^{\prime} 28.69^{\prime \prime} \mathrm{O}$ & & & \\
\hline \multirow[t]{2}{*}{ SV18 } & $18 / 08 / 2018$ & 1 & $36^{\circ} 36^{\prime} 57.73^{\prime \prime} \mathrm{S}$ & Santa Rosa & Espinal & Eco-Registros \\
\hline & & & $64^{\circ} 19^{\prime} 1.38^{\prime \prime O}$ & & & \\
\hline \multirow[t]{2}{*}{ SV19 } & $12 / 08 / 2018$ & 1 & $35^{\circ} 52^{\prime} 22.27^{\prime \prime} \mathrm{S}$ & Eduardo Castex & Pampa & Eco-Registros \\
\hline & & & $64^{\circ} 11^{\prime} 41.23^{\prime \prime O}$ & & & \\
\hline \multirow[t]{2}{*}{ SV20 } & $06 / 07 / 2018$ & 1 & $35^{\circ} 2^{\prime} 13.98^{\prime \prime} \mathrm{S}$ & Realicó & Pampa & Eco-Registros \\
\hline & & & $64^{\circ} 14^{\prime} 34.56^{\prime \prime O}$ & & & \\
\hline SV21 & $02 / 01 / 2018$ & 1 & $36^{\circ} 18^{\prime} 40.09^{\prime \prime S}$ & Winifreda & Pampa & Eco-Registros \\
\hline & & & $64^{\circ} 16^{\prime} 41.09^{\prime \prime O}$ & & & \\
\hline SV22 & $02 / 01 / 2018$ & 1 & $36^{\circ} 18^{\prime} 40.09^{\prime \prime} \mathrm{S}$ & Winifreda & Pampa & Eco-Registros \\
\hline & & & $64^{\circ} 16^{\prime} 41.09^{\prime \prime O}$ & & & \\
\hline SV23 & $10 / 10 / 2017$ & 1 & $35^{\circ} 39^{\prime} 41.33^{\prime \prime} \mathrm{S}$ & General Pico & Pampa & Eco-Registros \\
\hline & & & $63^{\circ} 45^{\prime} 0.60^{\prime \prime} \mathrm{O}$ & & & \\
\hline
\end{tabular}


Apéndice 1 (cont.).

Appendix 1 (cont.).

\begin{tabular}{|c|c|c|c|c|c|c|}
\hline ID & $\begin{array}{l}\text { Fecha } \\
\text { registro }\end{array}$ & $\mathrm{n}$ & $\begin{array}{l}\text { Coordenadas } \\
\text { geográficas }\end{array}$ & $\begin{array}{l}\text { Localidad } \\
\text { más cercana }\end{array}$ & $\begin{array}{l}\text { Eco- } \\
\text { región }\end{array}$ & Fuente \\
\hline SV24 & $23 / 09 / 2017$ & 1 & $\begin{array}{l}35^{\circ} 54^{\prime} 55.91^{\prime \prime S} \\
64^{\circ} 18^{\prime} 26.83^{\prime \prime O}\end{array}$ & Eduardo Castex & Pampa & Eco-Registros \\
\hline SV25 & $27 / 07 / 2017$ & 1 & $\begin{array}{l}35^{\circ} 40^{\prime} 49.87^{\prime \prime S} \mathrm{~S} \\
63^{\circ} 42^{\prime} 0.67^{\prime \prime O}\end{array}$ & General Pico & Pampa & Eco-Registros \\
\hline SV26 & $12 / 11 / 2016$ & 1 & $\begin{array}{l}35^{\circ} 54^{\prime} 42.00^{\prime \prime S} \\
64^{\circ} 15^{\prime} 7.82^{\prime \prime O}\end{array}$ & Eduardo Castex & Pampa & Eco-Registros \\
\hline SV27 & $28 / 08 / 2016$ & 1 & $\begin{array}{l}35^{\circ} 54^{\prime} 12.87^{\prime \prime S} \\
64^{\circ} 16^{\prime} 2.59^{\prime \prime} \mathrm{O}\end{array}$ & Eduardo Castex & Pampa & Eco-Registros \\
\hline SV28 & $13 / 11 / 2015$ & 1 & $\begin{array}{l}35^{\circ} 54^{\prime} 11.14^{\prime \prime S} \\
64^{\circ} 15^{\prime} 34.61^{\prime \prime O}\end{array}$ & Eduardo Castex & Pampa & Eco-Registros \\
\hline SV29 & $08 / 11 / 2015$ & 1 & $\begin{array}{l}36^{\circ} 38^{\prime} 58.61^{\prime \prime S} \\
64^{\circ} 20^{\prime} 17.48^{\prime \prime} \mathrm{O}\end{array}$ & Toay & Espinal & Eco-Registros \\
\hline SV30 & $18 / 07 / 2015$ & 1 & $\begin{array}{l}37^{\circ} 37^{\prime} 16.00^{\prime \prime} \mathrm{S} \\
66^{\circ} 49^{\prime} 38.00^{\prime \prime} \mathrm{O}\end{array}$ & Ruta del desierto & Monte & Eco-Registros \\
\hline SV31 & $03 / 10 / 2014$ & 1 & $\begin{array}{c}35^{\circ} 41^{\prime} 2.37^{\prime \prime} \mathrm{S} \\
63^{\circ} 46^{\prime} 43.36^{\prime \prime O}\end{array}$ & General Pico & Pampa & Eco-Registros \\
\hline SV32 & 07/01/2017 & $\geq 200$ & $\begin{array}{l}35^{\circ} 13^{\prime} 47.30^{\prime \prime} \mathrm{S} \\
63^{\circ} 43^{\prime} 4.62^{\prime \prime O}\end{array}$ & Intendente Alvear & Pampa & Propio \\
\hline sV33 & $25 / 09 / 2019$ & 1 & $\begin{array}{c}35^{\circ} 48^{\prime} 46.22^{\prime \prime S} \\
64^{\circ} 9^{\prime} 0.94^{\prime \prime} \mathrm{O}\end{array}$ & Monte Nievas & Pampa & Propio \\
\hline SV34 & $25 / 09 / 2019$ & 1 & $\begin{array}{l}35^{\circ} 46^{\prime} 4.69^{\prime \prime} \mathrm{S} \\
64^{\circ} 0^{\prime} 47.74^{\prime \prime O}\end{array}$ & Trenel & Pampa & Propio \\
\hline SV35 & $27 / 10 / 2019$ & 1 & $\begin{array}{l}35^{\circ} 48^{\prime} 57.35^{\prime \prime S} \\
63^{\circ} 49^{\prime} 43.46^{\prime \prime O}\end{array}$ & Dorila & Pampa & Propio \\
\hline sV36 & $05 / 11 / 2019$ & 1 & $\begin{array}{l}36^{\circ} 10^{\prime} 33.89^{\prime \prime} \mathrm{S} \\
63^{\circ} 41^{\prime} 4.78^{\prime \prime O}\end{array}$ & Huelen & Pampa & Propio \\
\hline SV37 & $09 / 11 / 2019$ & 10 & $\begin{array}{l}36^{\circ} 31^{\prime} 16.18^{\prime \prime S} \\
63^{\circ} 59^{\prime} 11.29^{\prime \prime O}\end{array}$ & Anguil & Pampa & Propio \\
\hline SV38 & $16 / 11 / 2019$ & 1 & $\begin{array}{l}36^{\circ} 24^{\prime} 6.77^{\prime \prime S} \\
63^{\circ} 55^{\prime} 8.33^{\prime \prime O}\end{array}$ & Uriburu & Pampa & Propio \\
\hline SV39 & $30 / 11 / 2019$ & 1 & $\begin{array}{l}36^{\circ} 21^{\prime} 23.83^{\prime \prime S} \\
64^{\circ} 2^{\prime} 19.54^{\prime \prime O}\end{array}$ & Mauricio Mayer & Pampa & Propio \\
\hline SV40 & $02 / 10 / 2019$ & 3 & $\begin{array}{l}35^{\circ} 43^{\prime} 24.89^{\prime \prime S} \mathrm{~S} \\
64^{\circ} 8^{\prime} 55.36^{\prime \prime} \mathrm{O}\end{array}$ & Trenel & Pampa & Propio \\
\hline SV41 & $19 / 11 / 2019$ & 1 & $\begin{array}{c}36^{\circ} 36^{\prime} 57.73^{\prime \prime S} \mathrm{~S} \\
64^{\circ} 19^{\prime} 1.38^{\prime \prime O}\end{array}$ & Santa Rosa & Pampa & Eco-Registros \\
\hline SV42 & 07/09/2019 & 4 & $\begin{array}{c}36^{\circ} 37^{\prime} 8.20^{\prime \prime S} \mathrm{~S} \\
64^{\circ} 19^{\prime} 17.80^{\prime \prime} \mathrm{O}\end{array}$ & Santa Rosa & Pampa & Eco-Registros \\
\hline SV43 & $19 / 06 / 2019$ & 1 & $\begin{array}{l}35^{\circ} 40^{\prime} 45.12^{\prime \prime S} \\
63^{\circ} 41^{\prime} 51.49^{\prime \prime O}\end{array}$ & General Pico & Pampa & e-Bird \\
\hline SV44 & $19 / 10 / 2019$ & 3 & $\begin{array}{l}35^{\circ} 39^{\prime} 57.60^{\prime \prime} \mathrm{S} \\
63^{\circ} 45^{\prime} 21.60^{\prime \prime} \mathrm{O}\end{array}$ & General Pico & Pampa & e-Bird \\
\hline SV45 & $19 / 10 / 2019$ & 3 & $\begin{array}{l}35^{\circ} 40^{\prime} 37.20^{\prime \prime} \mathrm{S} \\
63^{\circ} 44^{\prime} 16.80^{\prime \prime} \mathrm{O}\end{array}$ & General Pico & Pampa & e-Bird \\
\hline SV46 & $06 / 04 / 2019$ & 9 & $\begin{array}{l}36^{\circ} 2^{\prime} 60.00^{\prime \prime} \mathrm{S} \\
63^{\circ} 34^{\prime} 1.20^{\prime \prime} \mathrm{O}\end{array}$ & Quemú Quemú & Pampa & e-Bird \\
\hline SV47 & $05 / 04 / 2019$ & 13 & $\begin{array}{l}36^{\circ} 2^{\prime} 27.91^{\prime \prime S} \\
63^{\circ} 34^{\prime} 7.89^{\prime \prime O}\end{array}$ & Quemú Quemú & Pampa & e-Bird \\
\hline
\end{tabular}

